Research Article

\title{
Efficient Segmentation of Lymphoblast in Acute Lymphocytic Leukemia
}

\author{
Syed Ijaz Ur Rahman $\mathbb{D}^{1},{ }^{1}$ Misbah Jadoon $\mathbb{D}^{2},{ }^{2}$ Sikandar Ali $\mathbb{D}^{3},{ }^{3,4}$ Hizbullah Khattak $\mathbb{D}{ }^{5}$ \\ and Jiwei Huang $\mathbb{D}^{3,4}$
}

${ }^{1}$ Department of Computer Science, Islamia College University Peshawar, Peshawar, Pakistan

${ }^{2}$ Department of Computer Science, Women University Swabi, Khyber Pakhtunkhwa, Swabi 23430, Pakistan

${ }^{3}$ Department of Computer Science and Technology, China University of Petroleum, Beijing 102249, China

${ }^{4}$ Beijing Key Lab of Petroleum Data Mining, China University of Petroleum, Beijing 102249, China

${ }^{5}$ Department of Information Technology, Hazara University Mansehra, Khyber Pakhtunkhwa, Pakistan

Correspondence should be addressed to Sikandar Ali; sikandar@cup.edu.cn and Jiwei Huang; huangjw@cup.edu.cn

Received 6 April 2021; Accepted 22 April 2021; Published 3 May 2021

Academic Editor: Shah Nazir

Copyright ( 2021 Syed Ijaz Ur Rahman et al. This is an open access article distributed under the Creative Commons Attribution License, which permits unrestricted use, distribution, and reproduction in any medium, provided the original work is properly cited.

\begin{abstract}
Microscopic examination of peripheral blood smears and bone marrow is the preliminary step for the diagnosis of several lifethreatening diseases. Acute lymphocytic leukemia (ALL) is the most common disease in children that also needs an early diagnosis for on-time treatment as it spreads rapidly in the blood and forms immature lymphocytes. This might cause death in some weeks if left untreated. Manual methods in clinical laboratory being applied for the diagnosis of these diseases are inefficient and expensive, and the results are less accurate. A computer-aided system is the need of the day in which the most important step is segmenting the region of interest in blood or bone marrow for the detection and cure of the diseases which is the most challenging task. This study aims to propose a simple threshold-based segmentation technique by processing the S component of the HSV color space to segment the lymphoblasts in the bone marrow images of ALL patients. The technique was applied to 230 RGB bone marrow images having all the three types of ALL, i.e., L1, L2, and L3 resulted in the overall accuracy of $96.8 \%$.
\end{abstract}

\section{Introduction}

Blood and its components are the key substances for the microscopic examination of most life threat diseases such as leukemia, HIV, anemia, tumor, cancer, and thalassemia [1-5]. Leukemia is the type of cancer starting in bone marrow from immature lymphocytes called the lymphoblasts and then spreads through the blood in all over the body. The on-time treatment has a good chance of recovery for the patient. Lymphoblasts are mainly present in the bone marrow or peripheral blood slides of a patient having acute lymphoblastic leukemia [6-8]. According to French American British (FAB) classification, ALL is subclassified as L1, L2, and L3 on the basis of morphological structures exhibited in Figure 1. L1 type consists of nucleus and may or may not have a basophilic cytoplasm and have a small size, L2 having irregular nucleus and having a cytoplasm. L3 is large in size and have vacuoles in the cytoplasm. To diagnose ALL, it is totally dependent on the detection and morphological examination of blast cells in bone marrow and peripheral blood images. This process is performed by the hematologists' manually in clinical laboratories, which is a slow process, and it also depends on the experience of the laboratory expert and his physical condition. Hence, to overcome the limitations of these manual processes, fast and automated system is the need for the diagnosis of these lives in danger diseases. For the designing of this automated system, the most essential part is the segmentation of the region of interest, and it is the most difficult task to be done as the whole system depends on it. The aim of this study is to segment the blasts from bone marrow images and to introduce an effective segmentation approach. This can 


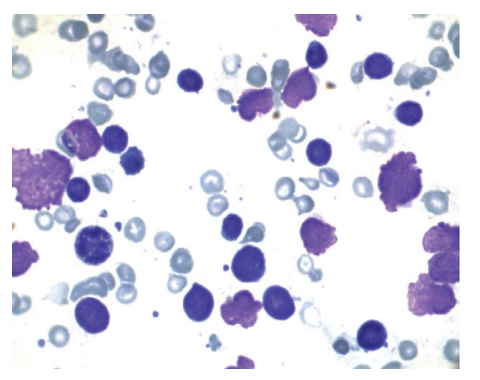

(a)

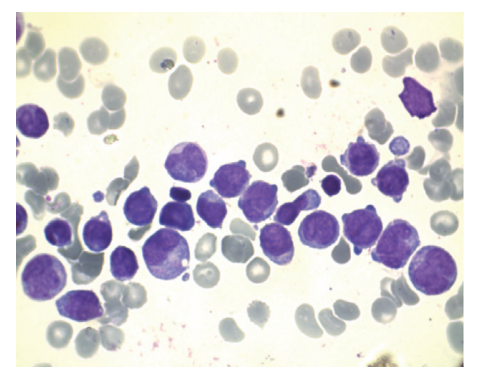

(b)

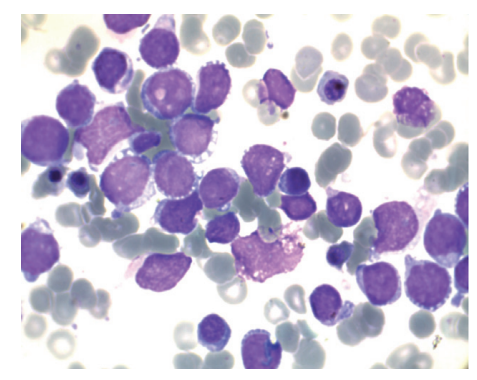

(c)

FIgURE 1: Three types of lymphoblast in acute lymphoblastic leukemia. (a) L1 blasts. (b) L2 blasts. (c) L3 blasts.

help the experts in the designing of the whole diagnostic system.

The further paper is organized into four main sections: Section 2 explores related work, Section 3 presents materials and methods reported in the paper, and Section 4 exhibits experimental results and discussion. Finally, Section 5 presents the conclusion.

\section{Related Studies}

There is no simple technique for the segmentation process as most of the researchers addressed this problem and reported their techniques in state of the art [5,9]. Sadeghian et al. [10] proposed a segmentation method for ALL blast in peripheral blood images for which canny edge detection algorithm was used with gradient vector flow GVF for the detection of the nucleus and then zack threshold was applied for cytoplasm. However, this method is applied to a single WBC image. Osowski et al. [11] recognized myoblasts in bone marrow by segmenting it and applied watershed transformation algorithm, but due to the crowded marrow, it resulted in oversegmentation. Madhloom et al. [12] proposed a method in which they used contrast stretching and arithmetic for the localization and segmenting white blood cells with 95\% accuracy; however, the method was used for the nucleus component, and that's why this is not identifying the leukemia cells as it required nucleus as well as the cytoplasm. In this study, we have proposed a method based on thresholding with the HSV color model, which could accurately segment the lymphoblasts for the detection of ALL in microscopic bone marrow images.

Tabrizi et al. [13] proposed snake contour for the segmentation of leukocytes; LVO, PCA algorithms, and SVM classifier is used for the identification. Mohamed and Far [14] segmentation was intensity based on Gram-Schmidt orthogonalization. Abbas and Mohamad [8] proposed a convolution mask along with Otsu method for the segmentation of nuclei of the lymphocytes for the detection of leukemia having the same limitation as faced by Madloom et al. [12] to detect leukemia nucleus, cytoplasm is needed. Mohapatra et al. [15] separated the interested region using $K$-means clustering and then used shadowed C-means (CSM) on the CIELAB color model for the segmentation of nuclei and cytoplasm of the leukocytes and then used an ensemble of classifiers for the classification. Adollah et al.
[16] reviewed different methods for the segmentation of leukocytes in which different authors addressed the problem with different techniques, i.e., Otsu threshold and circular histogram were used for the segmentation of WBCs, entropy with higher order is used as a feature, gray level threshold, filters with color matching, different morphological operators, shape information of binarizing and generating maximum intensity, watershed with seeds, and combining filters with scale-space were used for the segmentation purpose.

Comaniciu and Meer [17] recommend an image-guided decision support (IGDS) for the classification of different lymphomas and uses mean shift algorithm for the accurate segmentation; however, the system is not checked for acute leukemia. Abbas and Dzulkifli Mohamad [8] applied 2*2/6 convolution mask for repressing high values of RGB, and then Otsu method is applied to obtain nuclei, small areas were removed, and nuclei have been dilated for the required results of segmenting nuclei and detecting leukemia resulting in accuracy of $96.5 \%$.

Bhattacharjee and Saini [18] used a watershed algorithm for the segmentation of blast cells to detect ALL, and the segmentation process was followed with morphological operators, binary search tree, and Gaussian mixture model to classify the blasts. Pan et al. [19] employed extreme learning machine to segment the leukocytes on the basis of gradient threshold and entropy for the segmentation of multicolor objects on the HIS color model with Otsu's thresholding to properly segment leukocytes. Abbas et al. [6] applied a convolution filter of $3 \times 3$ on the red channel of the RGB image and then converted that image into the binary mask and on the analysis of the histogram, and after that, both the binary images were added which results in nuclei of leukocytes. Amin et al. [20] used K-means clustering to segment the lymphoblasts cells from the blood images. Geometric and statistical features were employed to classify that lymphoblast into subtypes of ALL using support vector machine (SVM).

Dhanachandra et al. [21] enhanced the contrast of the images for which the author used partial contrast stretching, then used subtractive clustering method followed by $K$ means clustering and calculating potential for each pixel, then finds the center cluster using Euclidean distance, and then reshapes the image. For noise removal, they use a median filter. Rawat et al. [22] proposed histogram equalization followed by global threshold and morphological 
opening to segment the nucleus of the blast cell and finally subtracted an image from the preprocessed image to obtain cytoplasm. To classify the images, geometrical, statistical, and chromatic features were extracted and fed to classifiers PCA-KNN, PCA-PNN, PCA-SVM, and PCA-ANFIS in hierarchical; this took a long time to classify the images. Nighat Bibi et al. [23] used dense convolution neural network and residual CNN with cloud framework for the detection and classification of all types of leukemia.

\section{Materials and Methods}

The main purpose of the segmentation process is to collect the lymphoblasts from the slides containing all the blood components. It will help in the detection and classification of acute leukemias [7]. The original images obtained from digital camera microscope are in the RGB color model. However, for speedy and accurate segmentation, it is converted to the HSV color model to process the $S$ component.

3.1. Image Database. Images for this study were taken in the department of hematology lab at Saidu Medical College, Swat, KPK, Pakistan, using Euromax digital camera microscope. The slides were stained with Giemsa under the supervision of Dr. Amreek Lal, and the images were taken with 100x lens and oil immersion. The total images of $L 1$ type 102, L2 type 120, L3 type 30 were kept in different folders classified according to (FAB) with the help of concerned hematologist.

3.2. Proposed Methodology. A simple method for the segmentation of lymphoblasts is proposed. The images were first converted to the HSV color model, and then the $S$ component is processed further to achieve good segmentation results. There are the subtypes of acute lymphoblastic leukemia according to the French American British classification, i.e., $L 1, L 2$, and $L 3$ having different morphologies [20]. So, we tested different threshold values for each class according to the morphological structures of the lymphoblast. In the $S$ component image, we found the maximum threshold value to find out the value ( $T_{r h}$ value) in which the blast could only segment, while the rest of the objects should be removed.

$$
\mathrm{TT}=\operatorname{Max}(\operatorname{Max}(S(i, j)))-\mathrm{T}_{r h} .
$$

Different values of $T_{r h}$ were checked with equation (1) for all classes and then take that value on which the lymphoblasts were segmented accurately. After this, a MATLAB command Imfill holes were applied to all the images to restore the lost information and then find out a seeded value of RGB color of the blast in the original input image to convert back the image into its original RGB color. The proposed research framework is exhibited in Figure 2.

3.3. Pseudocode. The proposed algorithm processed the image and segmented lymphoblast according to the following pseudocode: (i) Read the image $\operatorname{img}(i, j)$ from the directory in RGB color space

(ii) Convert RGB image $\operatorname{img}(i, j)$ to HSV color space img_seg $(i, j)$ for further processing

(iii) Get the $S$ component of the HSV color model, $S(i, j)$ as they have more information about blasts and segmentation occur easily

(iv) Find out the maximum threshold in $S(i, j)$ and then subtract that value on which other small objects remove and only the lymphoblasts remain

(v) If $S(i, j)$ is greater or equal to the threshold value, find out in the previous step and then give the value 1 , otherwise 0

(vi) For the lost information function, Imfill holes are used to fill the gaps and information

(vii) Wherever the value is 1 , convert to its original color in $\operatorname{RGB} \operatorname{img}(i, j)$

\section{Experimental Results}

Different $T_{r h}$ values were checked for the accurate segmentation in different experiments. Firstly, the value for L1 blasts was kept 0.40 but could not produce efficient results and then changed to 0.45 in which it is segmented accurately over 102 images. It has an irregular shape nucleus and in rare cases have a cytoplasm. For L2 blast, due to the morphology of having cytoplasm, we checked the value 0.47 and 0.48 , but the results were not satisfactory. On $\mathrm{T}_{\mathrm{rh}}$ value 0.50 , it produced good segmentation results. This was applied to 120 images having L2 morphology. L3 blasts are large in size and having vacuoles in the cytoplasm part of the cells for that the value was set higher than that of L2 0.52 to segment the blasts with vacuoles to turn out satisfactory results. After hole filling is applied to fill the gaps which occurred during the whole process, results are exhibited in Figures 3-5 for ALL subtypes L1, L2, and L3, respectively.

The segmentation results are shown in Figures 3-5 for lymphoblasts of types L1, L2, and L3, respectively, giving efficient results as compared to other methods. Performance accuracy of the technique used in our study is shown in Figure 6 .

4.1. Performance Analysis. The proposed technique was also compared in terms of segmentation accuracy for the evaluation with three different existing methods which is shown in Figure 7. It is used for the segmentation of five types of white blood cells, and those methods were proposed by Madloom et al. [12]; Tabrizi et al. [13]; and Mohamed and far [14] presented in Figure 7. The only limitation in this study is that sometimes it results in oversegmentation and sometimes the very important information is missing especially in the case of L3 subtype of the ALL which affects the overall accuracy. Further segmentation results of all the three types of lymphoblasts are shown in Figure 8. Finally Table 1 presents accuracy for each subtype. The algorithms were also checked with ground-truth data, and the result is shown in Table 2. 


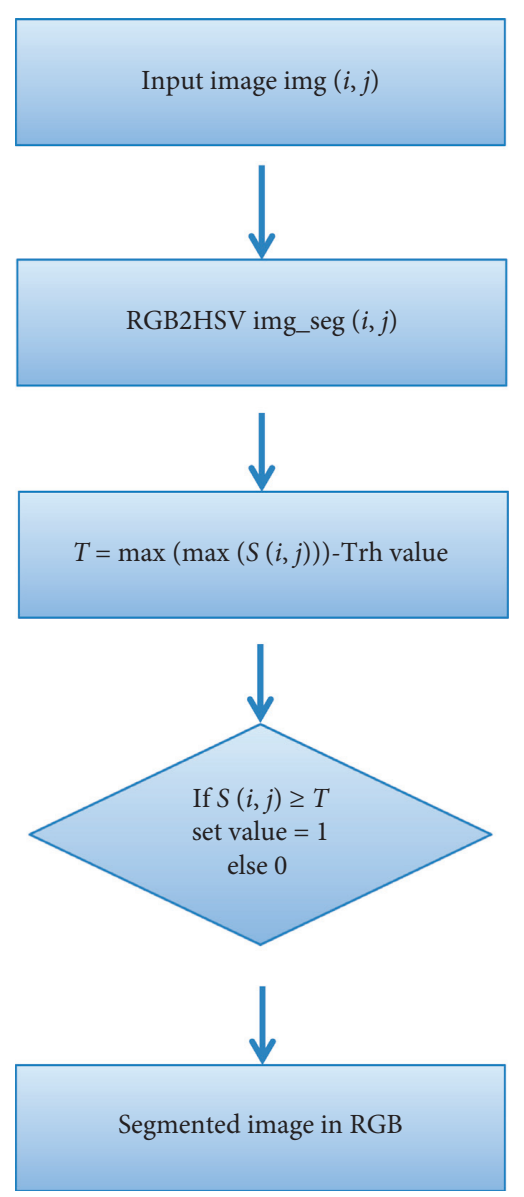

Figure 2: Proposed research framework.

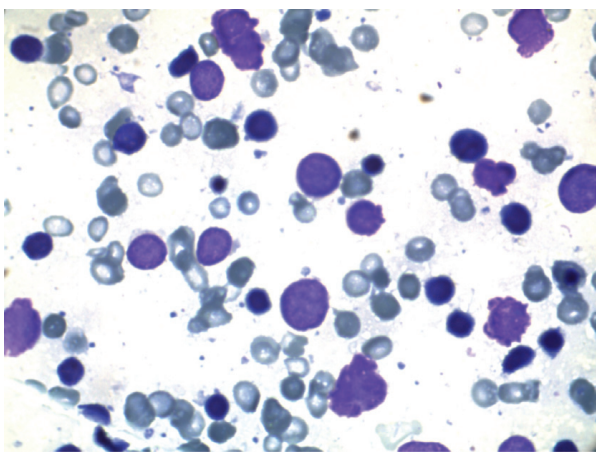

(a)

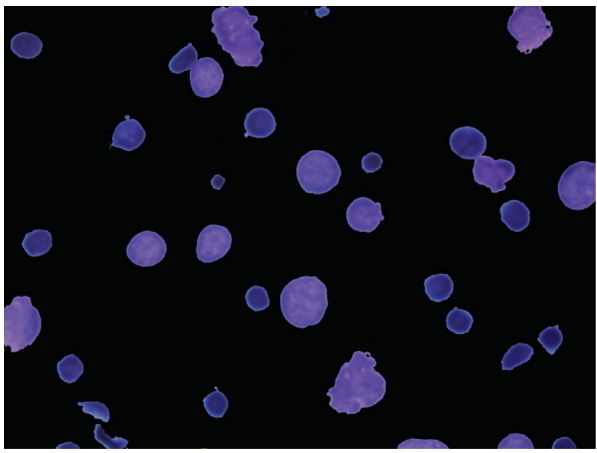

(b)

FIgURe 3: ALL subtype L1. (a) Original image. (b) Segmented lymphoblast.

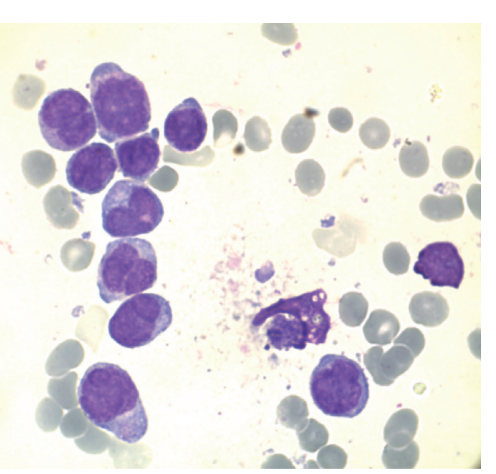

(a)

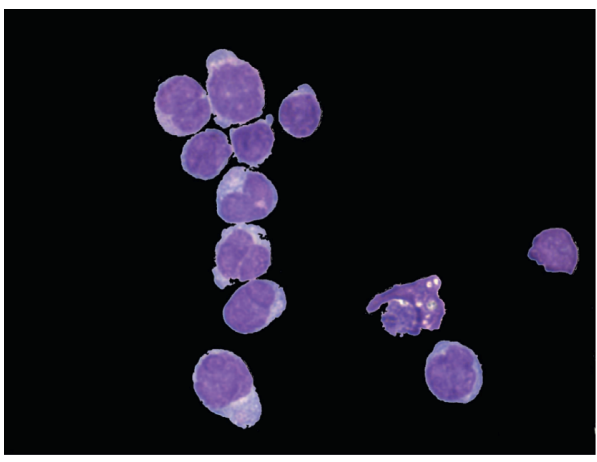

(b)

FIgURE 4: ALL subtype L2. (a) Original image. (b) Segmented lymphoblast. 


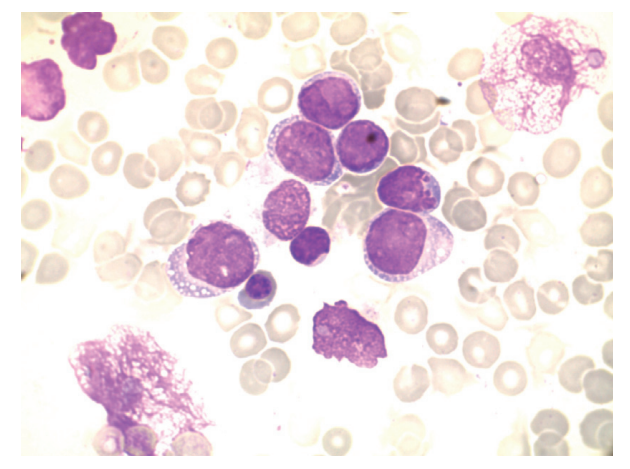

(a)

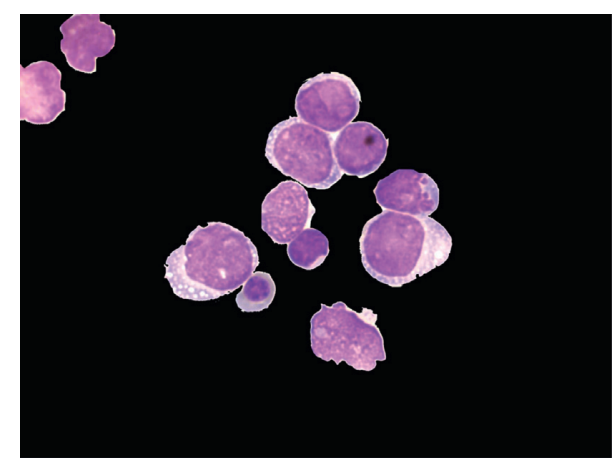

(b)

FIgure 5: ALL subtype L3. (a) Original image. (b) L3 segmented lymphoblast.

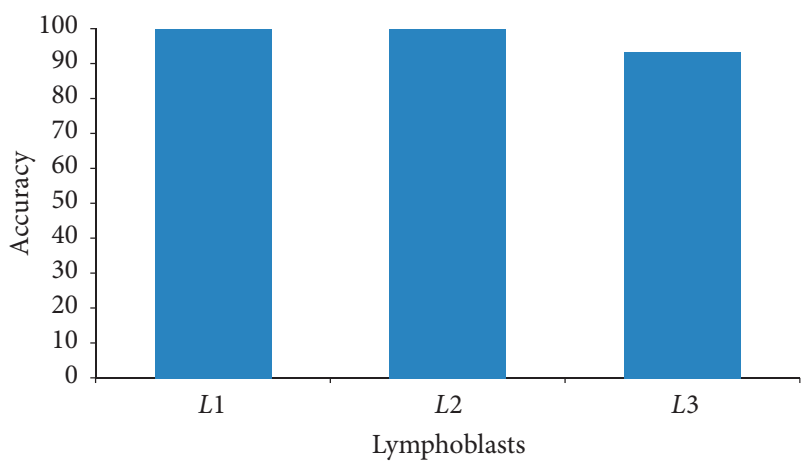

Figure 6: Performance accuracy of the proposed system.

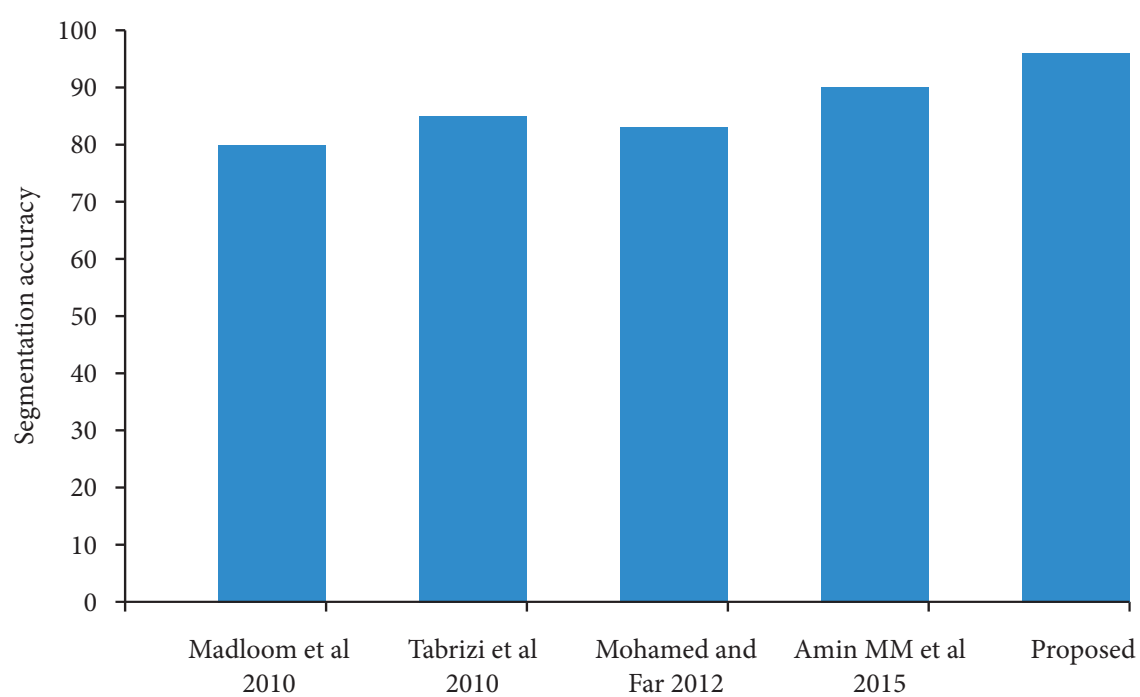

FIgURe 7: Performance evaluation of the proposed method with different techniques in state of art. 


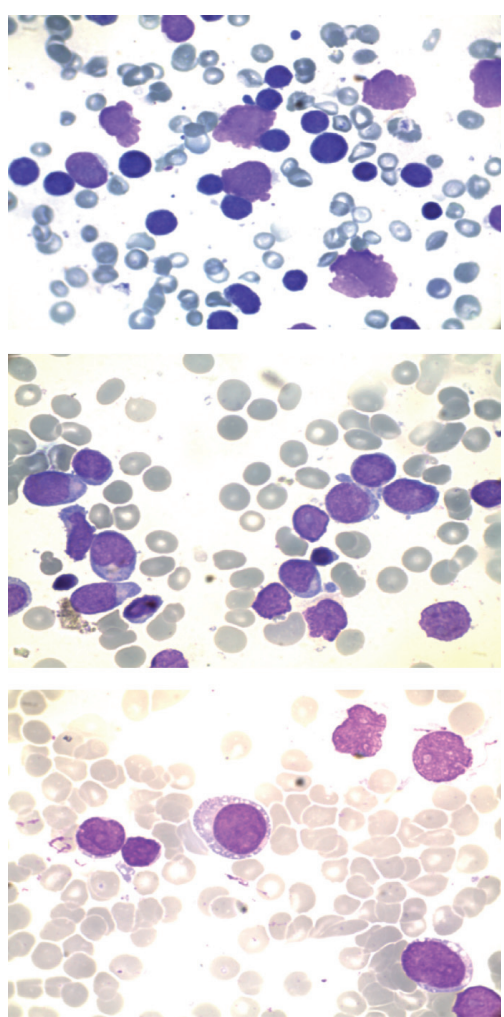

(a)
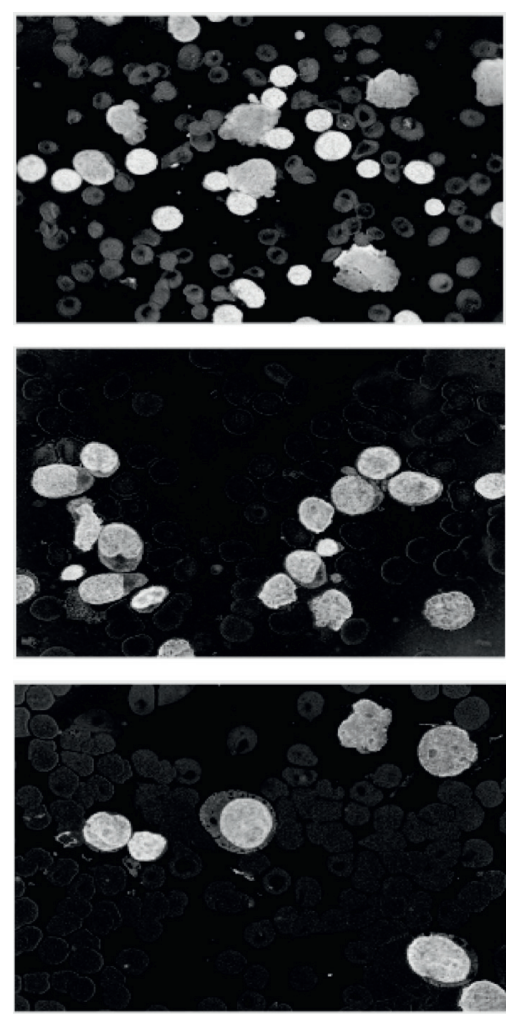

(b)
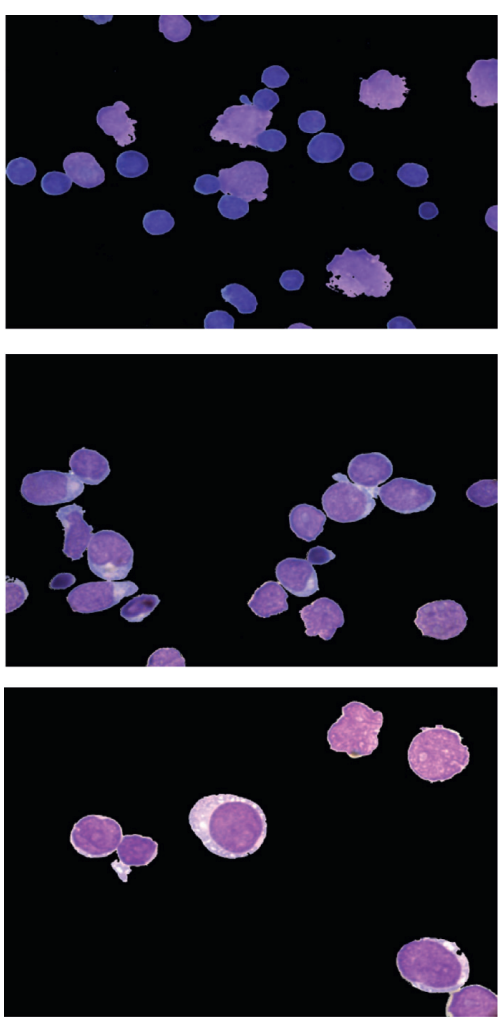

(c)

FIgurE 8: Experimental results. (a) Original images. (b) $S$ component of HSV color space. (c) Segmented images.

TABLE. 1: Accuracy for each subtype.

\begin{tabular}{lccc}
\hline ALL subtype & $L 1$ & $L 2$ & $L 3$ \\
\hline Total images & 102 & 120 & 30 \\
Accurate segmented & $102 / 102$ & $120 / 120$ & $28 / 30$ \\
Accuracy & $100 \%$ & $100 \%$ & $93.3 \%$ \\
\hline
\end{tabular}

TABLe 2: Proposed method evaluation with ground-truth data.

\begin{tabular}{lccc}
\hline Lymphoblast type & Recall & Precision & F-measure \\
\hline L1 & 0.9622 & 0.9811 & 0.9703211 \\
L2 & 0.9501 & 0.9712 & 0.96010211 \\
L3 & 0.9211 & 0.9322 & 0.9313221 \\
\hline
\end{tabular}

\section{Conclusion}

This paper has presented an effective approach to segment lymphoblast in a robust and accurate manner for the detection of leukemia by using simple threshold-based method with the HSV color model and attained an overall accuracy of $96.8 \%$. The proposed segmentation technique is easy to implement and then applied to 230 RGB images of bone marrow slides images of ALL patients. The segmentation accuracy for the subtypes L1 and L2 was 100\%; for the third subtype L3, the accuracy was $93.3 \%$ because of the vacuoles present in the cytoplasm area. Furthermore, the method is compared for performance evaluation with three other existing methods in terms of accuracy. We can also use the proposed method in general for the segmentation of blast cells in other types of leukemia. This is witnessed from our experimental results that the proposed method in this paper is more helpful in the segmentation of lymphoblasts specially for ALL. For future work, segmentation accuracy for L3 subtype of ALL could be improved and limitations will be covered.

\section{Data Availability}

The data that support the findings of this study are available from the corresponding author upon reasonable request.

\section{Additional Points}

Summary. Acute lymphocytic leukemia (ALL) is a crucial disease, and manual methods are inefficient and expensive. Automated system is proposed to identify ROI in blood/ marrow for the detection and cure of the disease by processing $S$ component of HSV of lymphoblast.

\section{Conflicts of Interest}

The authors declare no conflicts of interest.

\section{Acknowledgments}

This study was supported by the National Key Research and Development Plan (no. 2016YFC0303700), National Natural Science Foundation of China (no. 61972414), Beijing Natural Science Foundation (no. 4202066), Beijing Nova Program 
(no. Z201100006820082), and Fundamental Research Funds for Central Universities (nos. 2462020YJRC001 and 2462018YJRC040).

\section{References}

[1] S. Iqbal, M. U. Ghani, T. Saba, and A. Rehman, "Brain tumor segmentation in multi-spectral MRI using convolutional neural networks (CNN)," Microscopy Research and Technique, vol. 81, no. 4, pp. 419-427, 2018.

[2] S. Iqbal, M. Usman Ghani Khan, T. Saba, and A. Rehman, "Computer-assisted brain tumor type discrimination using magnetic resonance imaging features," Biomedical Engineering Letters, vol. 8, no. 1, pp. 5-28, 2017.

[3] A. Jamal, M. H. Alkawaz, A. Rehman, and T. Saba, "Retinal imaging analysis based on vessel detection," Microscopy Research and Technique, vol. 80, no. 7, pp. 799-811, 2017.

[4] B. Mughal, M. Sharif, N. Muhammad, and T. Saba, "A novel classification scheme to decline the mortality rate among women due to breast tumor," Microscopy Research and Technique, vol. 23, 2017.

[5] T. Saba, S. T. F. Bokhari, M. Sharif, M. Yasmin, and M. Raza, "Fundus image classification methods for the detection of glaucoma: a review," Microscopy Research and Technique, vol. 18, 2018.

[6] A. N. Mohamad, A. H. Abdullah, T. Al-Rodhaan, and M. AlDhelaan, "Nuclei segmentation of leukocytes in blood smear digital images," Pakistan Journal of Pharmaceutical Sciences, vol. 28, no. 5, pp. 1801-1806, 2015.

[7] A. Rehman, N. Abbas, T. Saba, S. Mehmood, and Z. Kolivand, "Classification of acute lymphoblastic leukemia using deep learning," Microscopy Research \& Technique, vol. 81, 2018.

[8] N. Abbas and D. Mohamad, "Automatic color nuclei segmentation of leukocytes for acute leukemia," Research Journal of Applied Sciences, Engineering and Technology, vol. 7, no. 14, pp. 2987-2993, 2014.

[9] A. Norouzi, M. S. M. Rahim, A. Altameem, T. Saba, A. E. Rada, and A. Rehman, "Medical image segmentation methods, algorithms," Applications IETE Technical Review, vol. 31, no. 3, pp. 199-213, 2014.

[10] F. Sadeghian, Z. Seman, A. R. Ramli, B. H. A. Kahar, and M. I. Saripan, "A framework for white blood cell segmentation in microscopic blood images using digital image processing," Biological Procedures Online, vol. 11, no. 1, p. 196, 2009.

[11] S. Osowski, T. Markiewicz, B. Marianska, and L. Moszczyński, "September. Feature generation for the cell image recognition of myelogenous leukemia," in Procedings of the Signal Processing Conference, 2004 12th European, IEEE, Vienna, Austria, September 2004.

[12] H. T. Madhloom, S. A. Kareem, H. Ariffin, A. A. Zaidan, H. O. Alanazi, and B. B. Zaidan, "An automated white blood cell nucleus localization and segmentation using image arithmetic and automatic threshold," Journal of Applied Sciences, vol. 10, no. 11, pp. 959-966, 2010.

[13] P. Tabrizi, S. Rezatofighi, and M. Yazdanpanah, "Using PCA and LVQ neural network for automatic recognition of five types of white blood cells," in Proceedings of the Annual International Conference of the IEEE Engineering in Medicine and Biology Society (EMBC, Berlin, Germany, August 2010.

[14] M. Mohamed and B. Far, "An enhanced threshold based technique for white blood cells nuclei automatic segmentation," in Proceeding of the IEEE 14th International Conference on E-Health Networking, pp. 202-207, Munich, Germany, September 2012.
[15] S. Mohapatra, D. Patra, and S. Satpathy, "An ensemble classifier system for early diagnosis of acute lymphoblastic leukemia in blood microscopic images," Neural Computing and Applications, vol. 24, no. 7-8, pp. 1887-1904, 2014.

[16] R. Adollah, M. Y. Mashor, N. M. Nasir, H. Rosline, H. Mahsin, and H. Adilah, "Blood cell image segmentation: a review," in Proceedings of the 4th Kuala Lumpur International Conference on Biomedical Engineering 2008, pp. 141-144, Kuala Lumpur, Malaysia, June 2008.

[17] D. Comaniciu and P. Meer, "Cell image segmentation for diagnostic pathology," in Advanced Algorithm Approaches to Medical Image Segmentation: State-of-The-Art Application in CardiologySpringer, Berlin, Germany, 2001.

[18] R. Bhattacharjee and L. M. Saini, "Detection of Acute Lymphoblastic Leukemia using watershed transformation technique," in Proceedings of the Signal Processing, Computing and Control (ISPCC), 2015 International Conference on 2015, pp. 383-386, Solan, India, September 2015.

[19] C. Pan, D. S. Park, Y. Yang, and H. M. Yoo, "Leukocyte image segmentation by visual attention and extreme learning machine," Neural Computing and Applications, vol. 21, no. 6, pp. 1217-1227, 2012.

[20] M. M. Amin, S. Kermani, A. Talebi, and M. G. Oghli, "Recognition of acute lymphoblastic leukemia cells in microscopic images using $\mathrm{k}$-means clustering and support vector machine classifier," Journal of Medical Signals and Sensors, vol. 5, no. 1, pp. 49-58, 2015.

[21] N. Dhanachandra, K. Manglem, and Y. J. Chanu, "Image segmentation using $\mathrm{K}$-means clustering algorithm and subtractive clustering algorithm," Procedia Computer Science, vol. 54, pp. 764-771, 2015.

[22] J. Rawat, A. Singh, H. S. Bhadauria, J. Virmani, and J. S. Devgun, "Classification of acute lymphoblastic leukaemia using hybrid hierarchical classifiers," Multimedia Tools and Applications, vol. 76, no. 18, pp. 19057-19085, 2017.

[23] N. Bibi, M. Sikandar, I. Ud Din, A. Almogren, and S. Ali, "IoMT-based automated detection and classification of leukemia using deep learning," Journal of Healthcare Engineering, vol. 12, pp. 1-12, 2020. 